\title{
A Boundary Value Problem for Bihypermonogenic Functions in Clifford Analysis
}

\author{
Xiaoli Bian $^{1}$ and Yuying Qiao ${ }^{2}$ \\ ${ }^{1}$ School of Science, Tianjin University of Technology and Education, Tianjin 300222, China \\ ${ }^{2}$ College of Mathematics and Information, Hebei Normal University, Shijiazhuang 050016, China \\ Correspondence should be addressed to Xiaoli Bian; bianxiaoli@yeah.net
}

Received 8 May 2014; Revised 25 June 2014; Accepted 1 July 2014; Published 20 July 2014

Academic Editor: Guo-Cheng Wu

Copyright (c) 2014 X. Bian and Y. Qiao. This is an open access article distributed under the Creative Commons Attribution License, which permits unrestricted use, distribution, and reproduction in any medium, provided the original work is properly cited.

\begin{abstract}
This paper deals with a nonlinear boundary value problem for bihypermonogenic functions in Clifford analysis. The integrals of quasi-Cauchy's type and Plemelj formula for bihypermonogenic functions are firstly reviewed briefly. The nonlinear Riemmann boundary value problem for bihypermonogenic functions is discussed and the existence of solutions is obtained, which also indicates that the linear boundary value problem has a unique solution.
\end{abstract}

\section{Introduction}

Clifford algebra is an associative and noncommutative algebraic structure that was set up at the beginning of the twentieth century. Clifford analysis is an important branch of modern analysis, which studies the functions defined in $\mathbb{R}^{n+1}$ with the value in Clifford algebra space [1]. Clifford analysis possesses not only important theoretical value but also applicable value, which plays an important role in many fields, such as quantum mechanics, Maxwell equation, and YangMills field. Since 1987, Xu [2, 3], Wen [4], Huang [5, 6], Qiao [7-9], and so forth have done a lot of work on boundary value problems for monogenic functions and biregular functions in Clifford analysis. Eriksson and Leutwiler [10-12] introduced hypermonogenic functions in Clifford analysis, studied some of its properties, and discussed the integral representation for hypermonogenic functions. Qiao [9] investigated the boundary value problems of hypermonogenic functions. In recent years, Zhang and $\mathrm{Du}[13,14]$ discussed Riemann boundary value problems and singular integral equations in Clifford analysis. Bian et al. [15] obtained the integral formulas and Plemelj formula for bihypermonogenic functions. Yang et al. [16] studied a kind of boundary value problem for hypermonogenic function vector. Zhang and Gürlebeck [17] studied Riemann boundary value problems in Clifford analysis.
In this paper, based on the integral formulas and Plemelj formula for bihypermonogenic functions presented in [15], we study a nonlinear Riemann boundary value problem for bihypermongenic functions. We first review briefly the integrals of quasi-Cauchy's type and Plemelj formula for bihypermonogenic functions and then prove the existence of solutions of a nonlinear Riemann boundary value problem and derive the unique solution of the corresponding linear Riemann boundary value problem.

\section{Preliminaries}

Let $C \ell_{0, n}$ be a real Clifford algebra over an $n+1$ dimensional real vector space $R^{n+1}$ with orthogonal basis $e:=$ $\left\{e_{0}, e_{1}, \ldots, e_{n}\right\}$, satisfying the relation $e_{i} e_{j}+e_{j} e_{i}=-2 \delta_{i j}(i, j=$ $1, \ldots, n)$, where $\delta_{i j}$ is the usual Kronecker delta. Then $C \ell_{0, n}$ has its basis $e_{0}=1, e_{1}, \ldots, e_{n} ; e_{1} e_{2}, \ldots, e_{n-1} e_{n} ; \ldots ; e_{1} \cdots e_{n}$. Hence the real Clifford algebra is formed by the elements presented as $a=\sum_{A} x_{A} e_{A}, x_{A} \in \mathbb{R}$, where $A=\left\{i_{1}, i_{2}, \ldots, i_{k} \mid\right.$ $\left.1 \leq i_{1}<i_{2}<\cdots<i_{k} \leq n\right\}$ or $A=\emptyset$ and $e_{\emptyset}=e_{0}$.

For $a \in C l_{0, n}$, we give some calculations as follows:

$$
a^{\prime}=\sum_{A} x_{A} e_{A}^{\prime}
$$


where $e_{A}^{\prime}=(-1)^{|A|} e_{A}$ and $|A|=n_{A}$ is the cardinality of $A$; that is, when $A=\emptyset,|A|=0$ and when $A=\left\{\alpha_{1}, \alpha_{2}, \ldots, \alpha_{h}\right\} \neq \emptyset$, then $|A|=h ; e_{0}^{\prime}=1, e_{i}^{\prime}=-e_{i}, i=1, \ldots, n$.

Recall that any element $x \in C e_{0, n}$ may be uniquely decomposed as $x=b+c e_{n}$, for $b, c \in C \ell_{0, n-1}$ (the Clifford algebra generated by $\left.e_{0}, \ldots, e_{n-1}\right)$. Using this decomposition, we define the mappings $P: C \ell_{0, n} \rightarrow C \ell_{0, n-1}$ and $Q: C \ell_{0, n} \rightarrow$ $C \ell_{0, n-1}$ by $P x=b$ and $Q x=c$. Note that if $x=\sum_{A} x_{A} e_{A} \in$ $C \ell_{0, n}$, then

$$
\begin{array}{rlrl}
P x & =\sum_{n \notin A} x_{A} e_{A}, & Q x=\sum_{n \in A} x_{A} e_{A \backslash\{n\}}, \\
P^{\prime} x=\sum_{n \notin A} x_{A} e_{A}^{\prime}, & Q^{\prime} x=\sum_{n \in A} x_{A} e_{A \backslash\{n\}}^{\prime} .
\end{array}
$$

We also introduce the Dirac operator

$$
D_{l} f=\sum_{i=0}^{n} e_{i} \frac{\partial f}{\partial x_{i}}, \quad D_{r} f=\sum_{i=0}^{n} \frac{\partial f}{\partial x_{i}} e_{i},
$$

and the modified Dirac operator

$$
\begin{aligned}
& M^{l} f(x)=D_{l} f(x)+(n-1) \frac{Q^{\prime} f}{x_{n}}, \\
& M^{r} f(x)=D_{r} f(x)+(n-1) \frac{Q f}{x_{n}} .
\end{aligned}
$$

Denote by $\Omega=\Omega_{1} \times \Omega_{2}$ an open connected set in the Euclidean space $\mathbb{R}^{m+1} \times \mathbb{R}^{k+1}, 1 \leq m \leq n, 1 \leq k \leq n$. Define a set $\mathscr{F}_{\Omega}^{(r)}$ to consist of all functions

$$
f(x, y)=\sum_{A \subset\{1, \ldots, m\} B \subset\{m+1, \ldots, m+k\}} f_{A, B}(x, y) e_{A} e_{B},
$$

with values in $C \ell_{0, k+m}$ for which $f_{A, B}(x, y) \in \mathscr{C}^{r}(\Omega)$.

Definition 1. Let $f \in \mathscr{F}_{\Omega}^{(1)}$ and $x \in \mathbb{R}^{m+1} \backslash\left\{x_{m}=0\right\}, y \in$ $\mathbb{R}^{k+1} \backslash\left\{y_{k}=0\right\}$. A function $f(x, y)$ is called bihypermonogenic on $\Omega$, if

$$
M_{x}^{l} f(x, y)=0, \quad M_{y}^{r} f(x, y)=0,
$$

for any $(x, y) \in \Omega$, where

$$
\begin{aligned}
M_{x}^{l} f(x, y)= & \frac{\partial f}{\partial x_{0}}(x, y)+\sum_{i=1}^{m} e_{i} \frac{\partial f}{\partial x_{i}}(x, y) \\
& +(m-1) \frac{Q_{x}^{\prime}(f(x, y))}{x_{m}}
\end{aligned}
$$

is the left modified Dirac operator in $C \ell_{0, m}$ calculated with respect to $x \in \mathbb{R}^{m+1} \backslash\left\{x_{m}=0\right\}$ and

$$
\begin{aligned}
M_{y}^{r} f(x, y)= & \frac{\partial f}{\partial y_{0}}(x, y)+\sum_{i=1}^{k} \frac{\partial f}{\partial y_{i}}(x, y) e_{i+m} \\
& +(k-1) \frac{Q_{y}(f(x, y))}{y_{k}}
\end{aligned}
$$

is the right modified Dirac operator in the Clifford algebra generated by $e_{0}, e_{m+1}, \ldots, e_{m+k}$ calculated with respect to $y \in$ $\mathbb{R}^{k+1} \backslash\left\{y_{k}=0\right\}$, where

$$
\begin{aligned}
Q_{x}^{\prime} f(x, y) & =\sum_{m \in A} \sum_{B} f_{A, B}(x, y) e_{A \backslash\{m\}}^{\prime} e_{B} \\
& =\sum_{m \in A} \sum_{B}(-1)^{|A \backslash\{m\}|} f_{A, B}(x, y) e_{A \backslash\{m\}} e_{B}, \\
Q_{y} f(x, y) & =\sum_{A} \sum_{m+k \in B} f_{A, B}(x, y) e_{A} e_{B \backslash\{m+k\}} .
\end{aligned}
$$

\section{The Cauchy Integral Formula and Plemelj Formula}

In this section, we give some simple review on the Cauchy integral formula and Plemelj formula for bihypermonogenic functions obtained by us and presented in [15]. We first give some notations which will be used in the following analysis.

A function $f(x, y): \partial \Omega_{1} \times \partial \Omega_{2} \rightarrow C l_{0, n}$ is said to be Hölder continuous on $\partial \Omega_{1} \times \partial \Omega_{2}$, if $f(x, y)$ satisfies

$$
\begin{gathered}
\left|f\left(x_{1}, y_{1}\right)-f\left(x_{2}, y_{2}\right)\right| \leq M_{1}\left|\left(x_{1}, y_{1}\right)-\left(x_{2}, y_{2}\right)\right|^{\beta} \\
\left(x_{1}, y_{1}\right),\left(x_{2}, y_{2}\right) \in \partial \Omega_{1} \times \partial \Omega_{2}, \quad(0<\beta<1) .
\end{gathered}
$$

Denote by $H\left(\partial \Omega_{1} \times \partial \Omega_{2}, \beta\right)$ the set of all Hölder continuous functions on $\partial \Omega_{1} \times \partial \Omega_{2}$ with the index $\beta$. For any $f \in$ $H\left(\partial \Omega_{1} \times \partial \Omega_{2}, \beta\right)$, define the norm in $H\left(\partial \Omega_{1} \times \partial \Omega_{2}, \beta\right)$ as $\|f\|_{\beta}=C\left(f, \partial \Omega_{1} \times \partial \Omega_{2}\right)+H\left(f, \partial \Omega_{1} \times \partial \Omega_{2}, \beta\right)$, where

$$
\begin{gathered}
C\left(f, \partial \Omega_{1} \times \partial \Omega_{2}\right)=\sup _{\substack{\left(u_{i}, v_{i}\right) \in \partial \Omega_{1} \times \partial \Omega_{2} \\
\left(u_{1}, v_{1}\right) \neq\left(u_{2}, v_{2}\right)}} \frac{\left|f\left(u_{1}, v_{1}\right)-f\left(u_{2}, v_{2}\right)\right|}{\left|\left(u_{1}, v_{1}\right)-\left(u_{2}, v_{2}\right)\right|^{\beta}}, \\
H\left(f, \partial \Omega_{1} \times \partial \Omega_{2}, \beta\right)=\max _{(u, v) \in \partial \Omega_{1} \times \partial \Omega_{2}}|f(u, v)|, \\
f \in H\left(\partial \Omega_{1} \times \partial \Omega_{2}, \beta\right) .
\end{gathered}
$$

Furthermore, for any $f, g \in H\left(\partial \Omega_{1} \times \partial \Omega_{2}, \beta\right)$, we have

$$
\|f g\|_{\beta} \leq J_{0}\|f\|_{\beta}\|g\|_{\beta} .
$$

Theorem 2 (see [15]). Let $\Omega^{\prime}$ and $\Omega^{\prime \prime}$ be open subsets of $\mathbb{R}_{+}^{m+1}$ and $\mathbb{R}_{+}^{k+1}$, respectively. Suppose that $\Omega_{1}$ and $\Omega_{2}$ satisfy $\overline{\Omega_{1}} \subset \Omega^{\prime}$ and $\overline{\Omega_{2}} \subset \Omega^{\prime \prime}$, respectively. The boundaries $\partial \Omega_{1}, \partial \Omega_{2}$ of $\Omega_{1}$, $\Omega_{2}$ are differentiable, oriented, compact Liapunov surfaces. If 
$\varphi(x, y)$ is a bihypermonogenic function in $\Omega^{\prime} \times \Omega^{\prime \prime}, x \in \Omega_{1}, y \in$ $\Omega_{2}$, then

$$
\begin{aligned}
\varphi(x, y) & \\
= & \lambda \int_{\partial \Omega_{1} \times \partial \Omega_{2}} E_{m}(u, x) d \sigma_{m}(u) \varphi(u, v) d \sigma_{k}(v) \mathbf{E}_{k}(v, y) \\
& -\lambda \int_{\partial \Omega_{1} \times \partial \Omega_{2}} E_{m}(u, x) d \sigma_{m}(u) \widetilde{\varphi(u, v)} \widetilde{d \sigma_{k}(v)} \mathbf{F}_{k}(v, y) \\
& -\lambda \int_{\partial \Omega_{1} \times \partial \Omega_{2}} F_{m}(u, x) \widetilde{d \sigma_{m}(u)} \widehat{\varphi(u, v)} d \boldsymbol{\sigma}_{k}(v) \mathbf{E}_{k}(v, y) \\
& +\lambda \int_{\partial \Omega_{1} \times \partial \Omega_{2}} F_{m}(u, x) \widetilde{d \sigma_{m}(u)} \widetilde{\widehat{\varphi(u, v)}} \widetilde{d \sigma_{k}(v)} \mathbf{F}_{k}(v, y),
\end{aligned}
$$

where

$$
\begin{aligned}
& d \sigma_{m}=d x_{1} \wedge d x_{2} \wedge \cdots d x_{m} \\
& +\sum_{i=1}^{m}(-1)^{i} e_{i} d x_{0} \wedge \cdots \wedge d x_{i-1} \wedge d x_{i+1} \wedge \cdots d x_{m}, \\
& d \boldsymbol{\sigma}_{k}=d y_{1} \wedge d y_{2} \wedge \cdots d y_{k} \\
& +\sum_{i=1}^{k}(-1)^{i} e_{i+m} d y_{0} \wedge d y_{1} \wedge \cdots \wedge d y_{i-1} \wedge d y_{i+1} \\
& \wedge \cdots d y_{k} \\
& v=v_{0}+v_{1} e_{m+1}+\cdots+v_{k} e_{m+k}, \\
& y=y_{0}+y_{1} e_{m+1}+\cdots+y_{k} e_{m+k} \text {, } \\
& \lambda=\frac{2^{m-1} x_{m}^{m-1} 2^{k-1} y_{k}^{k-1}}{\omega_{m+1} \omega_{k+1}}, \\
& E_{l}(u, x)=\frac{(u-x)^{-1}}{|u-x|^{l-1}|u-\widehat{x}|^{l-1}}, \quad u, x \in \mathbb{R}^{m+1} \text {, } \\
& F_{l}(u, x)=\frac{(\widehat{u}-x)^{-1}}{|u-x|^{l-1}|u-\widehat{x}|^{l-1}}, \quad u, x \in \mathbb{R}^{m+1}, \\
& \mathbf{E}_{l}(v, y)=\frac{(v-y)^{-1}}{|v-y|^{l-1}|v-\tilde{y}|^{l-1}}, \\
& \mathbf{F}_{l}(v, y)=\frac{(\widetilde{v}-y)^{-1}}{|v-y|^{l-1}|v-\tilde{y}|^{l-1}},
\end{aligned}
$$

and the involutions - and are defined by

$$
\begin{aligned}
& \widehat{e_{i}}=e_{i}, \quad i \in\{0,1, \ldots, m+k\} \backslash\{m\}, \\
& \widehat{e_{m}}=-e_{m}, \quad \widehat{a b}=\widehat{a} \widehat{b}, \\
& \widetilde{e}_{i}=e_{i}, \quad i \in\{0,1, \ldots, m+k-1\}, \\
& \widetilde{e_{m+k}}=-e_{m+k}, \quad \widetilde{a b}=\tilde{a} \tilde{b} .
\end{aligned}
$$

Definition 3 (see [15]). The integral

$$
\begin{aligned}
\phi\left(t_{1}, t_{2}\right) & \\
= & \lambda \int_{\partial \Omega_{1} \times \partial \Omega_{2}} E_{m}\left(u, t_{1}\right) d \sigma_{m}(u) \varphi(u, v) d \boldsymbol{\sigma}_{k}(v) \mathbf{E}_{k}\left(v, t_{2}\right) \\
& -\lambda \int_{\partial \Omega_{1} \times \partial \Omega_{2}} E_{m}\left(u, t_{1}\right) d \sigma_{m}(u) \widetilde{\varphi(u, v)} \widetilde{\mathbf{d} \sigma_{k}(v)} \mathbf{F}_{k}\left(v, t_{2}\right) \\
& -\lambda \int_{\partial \Omega_{1} \times \partial \Omega_{2}} F_{m}\left(u, t_{1}\right) \widetilde{d \sigma_{m}(u)} \widehat{\varphi(u, v)} d \sigma_{k}(v) \mathbf{E}_{k}\left(v, t_{2}\right) \\
& +\lambda \int_{\partial \Omega_{1} \times \partial \Omega_{2}} F_{m}\left(u, t_{1}\right) \widetilde{d \sigma_{m}(u)} \widetilde{\widehat{\varphi(u, v)}} \widetilde{d \sigma_{k}(v)} \mathbf{F}_{k}\left(v, t_{2}\right)
\end{aligned}
$$

is called a singular integral on $\partial \Omega_{1} \times \partial \Omega_{2}$, where $\lambda, E_{m}\left(u, t_{1}\right)$, $\mathbf{E}_{k}\left(v, t_{2}\right), F_{m}\left(u, t_{1}\right)$, and $\mathbf{F}_{k}\left(v, t_{1}\right)$ are given in Theorem 2.

Definition 4 (see [15]). Let $\delta>0$ be a constant and $\lambda_{\delta}=$ $B_{1}\left(t_{1}, \delta\right) \times B_{2}\left(t_{2}, \delta\right)$, where $B_{i}\left(t_{i}, \delta\right)(i=1,2)$ are balls with the center at $t_{i}$ and the radius $\delta>0$. Denote

$$
\begin{aligned}
& \phi_{\delta}\left(t_{1}, t_{2}\right) \\
& =\lambda \int_{\partial \Omega_{1} \times \partial \Omega_{2} \backslash \lambda_{\delta}} E_{m}\left(u, t_{1}\right) d \sigma_{m}(u) \varphi(u, v) d \boldsymbol{\sigma}_{k}(v) \mathbf{E}_{k}\left(v, t_{2}\right) \\
& -\lambda \int_{\partial \Omega_{1} \times \partial \Omega_{2} \backslash \lambda_{\delta}} E_{m}\left(u, t_{1}\right) d \sigma_{m}(u) \widetilde{\varphi(u, v)} \widetilde{\mathbf{d} \sigma_{k}(v)} \mathbf{F}_{k}\left(v, t_{2}\right) \\
& -\lambda \int_{\partial \Omega_{1} \times \partial \Omega_{2} \backslash \lambda_{\delta}} F_{m}\left(u, t_{1}\right) \widehat{d \sigma_{m}(\mu)} \widehat{\varphi(u, v)} d \boldsymbol{\sigma}_{k}(v) \mathbf{E}_{k}\left(v, t_{2}\right) \\
& +\lambda \int_{\partial \Omega_{1} \times \partial \Omega_{2} \backslash \lambda_{\delta}} F_{m}\left(u, t_{1}\right) \widetilde{d \sigma_{m}(u)} \widetilde{\widehat{\varphi(u, v)}} \widetilde{\mathbf{d} \sigma_{k}(v)} \mathbf{F}_{k}\left(v, t_{2}\right) .
\end{aligned}
$$

If $\lim _{\delta \rightarrow 0} \phi_{\delta}\left(t_{1}, t_{2}\right)=I$, then $I$ is called the Cauchy principal value of a singular integral, denoted by $I=\phi\left(t_{1}, t_{2}\right)$.

Lemma 5 (see [11]). Let $\Omega$ be an open subset of $\mathbb{R}_{+}^{n+1}=\{x=$ $\left.\left(x_{0}, x_{1}, \ldots, x_{n}\right) \mid x_{n}>0\right\}$ and let $K$ be an $n+1$-chain satisfying $\bar{K} \subset \Omega$; then

$$
\begin{aligned}
& \frac{2^{n-1} y_{n}^{n-1}}{\omega_{n+1}}\left(\int_{\partial K} E_{n}(x, y) d \sigma(x)-\int_{\partial K} F_{n}(x, y) \overline{d \sigma(x)}\right) \\
& \quad= \begin{cases}1, & y \in K, \\
0, & y \in \mathbb{R}_{+}^{n+1}-\bar{K} .\end{cases}
\end{aligned}
$$

Lemma 6 (see [11]). Let $\Omega, K$ and $\partial K$ be as in Lemma 5 and $y \in \partial K$; then

$$
\frac{2^{n-1} y_{n}^{n-1}}{\omega_{n+1}}\left(\int_{\partial K} E_{n}(x, y) d \sigma(x)-\int_{\partial K} F_{n}(x, y) \overline{d \sigma(x)}\right)=\frac{1}{2} .
$$


Theorem 7 (see [15]). If $\varphi(u, v) \in H\left(\partial \Omega_{1} \times \partial \Omega_{2}, \beta\right)$, then there exists the Cauchy principal value of singular integrals and

$$
\begin{aligned}
\phi\left(t_{1}, t_{2}\right) & \\
= & -\frac{1}{4} \varphi\left(t_{1}, t_{2}\right)+X_{1}\left(t_{1}, t_{2}\right)+X_{2}\left(t_{1}, t_{2}\right)+X_{3}\left(t_{1}, t_{2}\right) \\
& +X_{4}\left(t_{1}, t_{2}\right)+\frac{1}{4}\left(P_{1} \varphi+P_{2} \varphi\right)+\frac{1}{4}\left(Q_{1} \varphi+Q_{2} \varphi\right),
\end{aligned}
$$

where

$$
\begin{aligned}
& X_{1}\left(t_{1}, t_{2}\right) \\
& =\lambda \int_{\partial \Omega_{1} \times \partial \Omega_{2}} E_{m}\left(u, t_{1}\right) d \sigma_{m}(u) \psi_{1}(u, v) d \boldsymbol{\sigma}_{k}(v) \\
& \times \mathbf{E}_{k}\left(v, t_{2}\right), \\
& X_{2}\left(t_{1}, t_{2}\right) \\
& =-\lambda \int_{\partial \Omega_{1} \times \partial \Omega_{2}} E_{m}\left(u, t_{1}\right) d \sigma_{m}(u) \psi_{2}(u, v) \widetilde{d \sigma_{k}(v)} \\
& \times \mathbf{F}_{k}\left(v, t_{2}\right), \\
& X_{3}\left(t_{1}, t_{2}\right) \\
& =-\lambda \int_{\partial \Omega_{1} \times \partial \Omega_{2}} F_{m}\left(u, t_{1}\right) \widehat{d \sigma_{m}(u)} \psi_{3}(u, v) d \sigma_{k}(v) \\
& \times \mathbf{E}_{k}\left(v, t_{2}\right), \\
& X_{4}\left(t_{1}, t_{2}\right) \\
& =\lambda \int_{\partial \Omega_{1} \times \partial \Omega_{2}} F_{m}\left(u, t_{1}\right) \widehat{d \sigma_{m}(u)} \psi_{4}(u, v) \widetilde{d \sigma_{k}(v)} \\
& \times \mathbf{F}_{k}\left(v, t_{2}\right),
\end{aligned}
$$

$\psi_{1}(u, v)=\varphi(u, v)-\varphi\left(u, t_{2}\right)-\varphi\left(t_{1}, v\right)+\varphi\left(t_{1}, t_{2}\right)$,

$\psi_{2}(u, v)=\widetilde{\varphi(u, v)}-\varphi\left(u, t_{2}\right)-\widetilde{\varphi\left(t_{1}, v\right)}+\varphi\left(t_{1}, t_{2}\right)$,

$\psi_{3}(u, v)=\widehat{\varphi(u, v)}-\widehat{\varphi\left(u, t_{2}\right)}-\varphi\left(t_{1}, v\right)+\varphi\left(t_{1}, t_{2}\right)$,

$\psi_{4}(u, v)=\widetilde{\widehat{\varphi(u, v)}}-\widehat{\varphi\left(u, t_{2}\right)}-\widetilde{\varphi\left(t_{1}, v\right)}+\varphi\left(t_{1}, t_{2}\right)$,

$P_{1} \varphi=2 \lambda_{1} \int_{\partial \Omega_{1}} E_{m}\left(u, t_{1}\right) d \sigma_{m}(u) \varphi\left(u, t_{2}\right)$,

$P_{2} \varphi=2 \lambda_{2} \int_{\partial \Omega_{2}} \varphi\left(t_{1}, v\right) d \boldsymbol{\sigma}_{k}(v) \mathbf{E}_{k}\left(v, t_{2}\right)$,

$Q_{1} \varphi=-2 \lambda_{1} \int_{\partial \Omega_{1}} F_{m}\left(u, t_{1}\right) \widehat{d \sigma_{m}(u)} \widehat{\varphi\left(u, t_{2}\right)}$,

$Q_{2} \varphi=-2 \lambda_{2} \int_{\partial \Omega_{2}} \widetilde{\varphi\left(t_{1}, v\right)} \widetilde{d \sigma_{k}(v)} \mathbf{F}_{k}\left(v, t_{2}\right)$,

$\lambda=\lambda_{1} \lambda_{2}, \quad \lambda_{1}=\frac{2^{m-1} x_{m}^{m-1}}{\omega_{m+1}}, \quad \lambda_{2}=\frac{2^{k-1} y_{k}^{k-1}}{\omega_{k+1}}$.
Set $\Omega_{i}^{+}=\Omega_{i}, \quad(i=1,2), \Omega_{1}^{-}=\mathbb{R}_{+}^{m+1} \backslash \overline{\Omega_{1}}, \Omega_{2}^{-}=\mathbb{R}_{+}^{k+1} \backslash \overline{\Omega_{2}}$, and denote $x\left(\in \Omega_{1}^{ \pm}\right) \rightarrow t_{1} \in \partial \Omega_{1}$ by $x \rightarrow t_{1}^{ \pm}$. Moreover denote $y\left(\in \Omega_{2}^{ \pm}\right) \rightarrow t_{2} \in \partial \Omega_{2}$ by $y \rightarrow t_{2}^{ \pm}$and denote by $\phi^{ \pm \pm}\left(t_{1}, t_{2}\right)$ the limits of $\phi(x, y)$ when $(x, y) \rightarrow\left(t_{1}^{ \pm}, t_{2}^{ \pm}\right)$. Then we have the following important theorem.

Theorem 8 (see [15]). If $\varphi(u, v) \in H\left(\partial \Omega_{1} \times \partial \Omega_{2}, \beta\right)$, then

$$
\begin{aligned}
& \phi^{++}\left(t_{1}, t_{2}\right) \\
& =\frac{1}{4}\left[\varphi\left(t_{1}, t_{2}\right)+P_{1}(\varphi)+P_{2}(\varphi)+Q_{1}(\varphi)+Q_{2}(\varphi)\right. \\
& \left.+P_{3}(\varphi)\right] \text {, } \\
& \phi^{+-}\left(t_{1}, t_{2}\right) \\
& =\frac{1}{4}\left[-\varphi\left(t_{1}, t_{2}\right)-P_{1}(\varphi)+P_{2}(\varphi)-Q_{1}(\varphi)+Q_{2}(\varphi)\right. \\
& \left.+P_{3}(\varphi)\right], \\
& \phi^{-+}\left(t_{1}, t_{2}\right) \\
& =\frac{1}{4}\left[-\varphi\left(t_{1}, t_{2}\right)+P_{1}(\varphi)-P_{2}(\varphi)+Q_{1}(\varphi)-Q_{2}(\varphi)\right. \\
& \left.+P_{3}(\varphi)\right], \\
& \phi^{--}\left(t_{1}, t_{2}\right) \\
& =\frac{1}{4}\left[\varphi\left(t_{1}, t_{2}\right)-P_{1}(\varphi)-P_{2}(\varphi)-Q_{1}(\varphi)-Q_{2}(\varphi)\right. \\
& \left.+P_{3}(\varphi)\right],
\end{aligned}
$$

where $\left(t_{1}, t_{2}\right) \in \partial \Omega_{1} \times \partial \Omega_{2}, P_{3}(\varphi)=4 \phi\left(t_{1}, t_{2}\right)$.

\section{The Boundary Value Problem for Bihypermonogenic Functions}

In this section, we consider the boundary value problem.

Definition 9. Let $\Omega_{1} \times \Omega_{2}$ and $H\left(\partial \Omega_{1} \times \partial \Omega_{2}, \beta\right)$ be as before. We want to find a bihypermonogenic function $\phi(x, y)$ defined in $\mathbb{R}_{+}^{m+1} \times \mathbb{R}_{+}^{k+1} / \partial \Omega_{1} \times \partial \Omega_{2}$, which is continuous to $\partial \Omega_{1} \times \partial \Omega_{2}$ and $\phi^{+-}(x, \infty)=\phi^{-+}(\infty, y)=\phi^{--}(\infty, \infty)=0$ and satisfies the nonlinear boundary condition

$$
\begin{gathered}
A\left(t_{1}, t_{2}\right) \phi^{++}\left(t_{1}, t_{2}\right)+B\left(t_{1}, t_{2}\right) \phi^{+-}\left(t_{1}, t_{2}\right) \\
+C\left(t_{1}, t_{2}\right) \phi^{-+}\left(t_{1}, t_{2}\right)+D\left(t_{1}, t_{2}\right) \phi^{--}\left(t_{1}, t_{2}\right) \\
=g\left(t_{1}, t_{2}\right) f\left(t_{1}, t_{2}, \phi^{++}\left(t_{1}, t_{2}\right), \phi^{+-}\left(t_{1}, t_{2}\right), \phi^{-+}\left(t_{1}, t_{2}\right),\right. \\
\left.\phi^{--}\left(t_{1}, t_{2}\right)\right),
\end{gathered}
$$

in which $A\left(t_{1}, t_{2}\right), B\left(t_{1}, t_{2}\right), C\left(t_{1}, t_{2}\right), D\left(t_{1}, t_{2}\right), g\left(t_{1}, t_{2}\right) \quad \epsilon$ $H\left(\partial \Omega_{1} \times \partial \Omega_{2}, \beta\right)$ and $f$ are known functions. The above boundary value problem is called Problem R. 
From Theorem 8 , we can transform the boundary condition of Problem R into an integral equation

$$
F \varphi=\varphi,
$$

where

$F \varphi$

$$
\begin{aligned}
= & (A+B)\left(\varphi+P_{1} \varphi+P_{2} \varphi+Q_{1} \varphi+Q_{2} \varphi+P_{3} \varphi\right) \\
& +(C+D)\left(-\varphi+P_{1} \varphi-P_{2} \varphi+Q_{1} \varphi-Q_{2} \varphi+P_{3} \varphi\right) \\
& +(B+D)\left(2 \varphi-2 P_{1} \varphi-2 Q_{1} \varphi\right)+(1-4 B) \varphi-4 g f
\end{aligned}
$$

Theorem 10 (see [9]). Let $\Omega, \partial \Omega \subset R_{+}^{l+1}$, and let $H(\partial \Omega, \beta)$ be the set of Hölder continuous functions on $\partial \Omega$ with the index $\beta$. For $\varphi \in H(\partial \Omega, \beta)$ and

$$
\begin{gathered}
\theta \varphi=\phi \varphi-\frac{\varphi}{2}, \\
\phi \varphi=\frac{2^{l-1} x_{l}^{l-1}}{\omega_{l+1}}\left[\int_{\partial \Omega} E_{l}(t, x) d \sigma_{0}(t) \varphi(t)\right. \\
\left.-F_{l}(t, x) \widehat{d \sigma_{0}(t)} \widehat{\varphi(t)}\right],
\end{gathered}
$$

where $E_{l}(t, x), F_{l}(t, x)$ are as before, then $\theta \varphi$ is a hypermonogenic function with

$$
\|\theta \varphi\|_{\beta} \leq J_{1}\|\varphi\|_{\beta},
$$

where $J_{1}$ is a constant independent of $\varphi$.

Lemma 11 (see [6]). Let $E_{m}(u, x), E_{m}\left(u, t_{1}\right)$ be the same as in Theorem 2. If $u \in \overline{\Omega_{1}}, x \in \overline{\Omega_{2}}, t_{1} \in \overline{\Omega_{2}}$, then there exists a constant $M$ such that

$$
\begin{aligned}
& \left|E_{m}(u, x)-E_{m}\left(u, t_{1}\right)\right| \\
& \quad \leq M\left[\sum_{i=0}^{m-1}\left|\frac{u-t_{1}}{u-x}\right|^{i}\left|\frac{x-t_{1}}{u-x}\right|+\left|x-t_{1}\right|\right]\left|u-t_{1}\right|^{-m} .
\end{aligned}
$$

Lemma 12. If $\varphi\left(t_{1}, t_{2}\right) \in H\left(\partial \Omega_{1} \times \partial \Omega_{2}, \beta\right)$, then

$$
\begin{aligned}
& \left\|\varphi \pm\left(P_{i} \varphi+Q_{i} \varphi\right)\right\|_{\beta} \leq J_{2}\|\varphi\|_{\beta}, \\
& \left\|P_{i} \varphi+Q_{i} \varphi\right\|_{\beta} \leq J_{2}\|\varphi\|_{\beta}, \quad i=1,2,
\end{aligned}
$$

where $\mathrm{J}_{2}$ is a positive constant.

Proof. Using the following equations:

$$
\begin{aligned}
P_{1} \varphi+Q_{1} \varphi= & 2 \lambda_{1} \int_{\partial \Omega_{1}} E_{m}\left(u, t_{1}\right) d \sigma_{m}(u) \varphi\left(\mu, t_{2}\right) \\
& -2 \lambda_{1} \int_{\partial \Omega_{1}} F_{m}\left(u, t_{1}\right) \widehat{d \sigma_{m}(u)} \widehat{\varphi\left(u, t_{2}\right)},
\end{aligned}
$$

$$
\begin{aligned}
P_{2} \varphi+Q_{2} \varphi= & 2 \lambda_{2} \int_{\partial \Omega_{2}} \varphi\left(t_{1}, v\right) d \sigma_{k}(v) E_{k}\left(v, t_{2}\right) \\
& -2 \lambda_{2} \int_{\partial \Omega_{2}} \widetilde{\varphi\left(t_{1}, v\right)} \widetilde{d \sigma_{k}(v)} F_{k}\left(v, t_{2}\right)
\end{aligned}
$$

and Theorem 10, we can obtain the result.

Theorem 13. Suppose the boundaries $\partial \Omega_{1}, \partial \Omega_{2}$ of $\Omega_{1}, \Omega_{2}$ be differentiable, oriented, compact Liapunov surfaces. If $\varphi\left(t_{1}, t_{2}\right) \in H\left(\partial \Omega_{1} \times \partial \Omega_{2}, \beta\right)$, then

$$
\left\|\left(P_{2} \varphi+Q_{2} \varphi\right) \pm P_{3} \varphi\right\|_{\beta} \leq J_{3}\|\varphi\|_{\beta},
$$

where $J_{3}$ is a positive constant which is independent of $\varphi$.

Proof. From (20), it follows that

$$
P_{2} \varphi+Q_{2} \varphi-P_{3} \varphi=\varphi-4 \sum_{i=1}^{4} X_{i}\left(t_{1}, t_{2}\right)-\left(P_{1} \varphi+Q_{1} \varphi\right)
$$

Moreover, based on Lemma 12 we only need to prove $\left\|\sum_{i=1}^{4} X_{i}\left(t_{1}, t_{2}\right)\right\|_{\beta} \leq J_{4}\|\varphi\|_{\beta}$. It is easy to prove $\left|\sum_{i=1}^{4} X_{i}\left(t_{1}, t_{2}\right)\right| \leq B_{1}\|\varphi\|_{\beta}$. We rewrite $\psi_{i}(u, v)$ as $\psi_{i}^{0}\left(t_{1}, t_{2}\right), \quad(i=1,2,3,4)$. Now we consider $H\left(\sum_{i=1}^{4} X_{i}\left(t_{1}, t_{2}\right), \partial \Omega_{1} \times \partial \Omega_{2}, \beta\right)$ and write $\delta=\mid\left(t_{1}, t_{2}\right)-$ $\left(t_{1}^{\prime}, t_{2}^{\prime}\right) \mid=\sqrt{\delta_{1}^{2}+\delta_{2}^{2}}$ for any $\left(t_{1}, t_{2}\right),\left(t_{1}^{\prime}, t_{2}^{\prime}\right) \in \partial \Omega_{1} \times \partial \Omega_{2}$ and denote by $\rho_{01}, \rho_{02}, \rho_{01}^{\prime}, \rho_{02}^{\prime}$ the projections of $\left|\mu-t_{1}\right|$, $\left|v-t_{2}\right|,\left|\mu-t_{1}^{\prime}\right|,\left|v-t_{2}^{\prime}\right|$ on the tangent plane of $t_{1}, t_{2}, t_{1}^{\prime}, t_{2}^{\prime}$, respectively. Moreover we construct spheres $O_{i}\left(t_{i}, 3 \delta_{i}\right)$ with the center at $t_{i}$ and radius $3 \delta_{i}$, where $6 \delta_{i}<d_{i}, \delta_{i}<1, i=1,2$, where $d_{i}$ is a constant as in [5]. Denote by $\partial \Omega_{i 1}, \partial \Omega_{i 2}$ the part of $\partial \Omega_{i}$ lying inside the sphere $O_{i}$ and its surplus part, respectively, and set

$$
\begin{aligned}
R\left(\partial \Omega_{1} \times \partial \Omega_{2}\right) & =\sum_{i=1}^{4} X_{i}\left(t_{1}, t_{2}\right)-\sum_{i=1}^{4} X_{i}\left(t_{1}^{\prime}, t_{2}^{\prime}\right) \\
& =\sum_{i=1}^{4} \overline{X_{i}}\left(\partial \Omega_{1} \times \partial \Omega_{2}\right)-\sum_{i=1}^{4} \overline{\overline{X_{i}}}\left(\partial \Omega_{1} \times \partial \Omega_{2}\right) .
\end{aligned}
$$

From

$$
\begin{gathered}
\left|\psi_{i}^{0}\left(t_{1}, t_{2}\right)\right| \leq M\|\varphi\|_{\beta}\left|u-t_{1}\right|^{\beta}, \\
\left|\psi_{i}^{0}\left(t_{1}, t_{2}\right)\right| \leq M\|\varphi\|_{\beta}\left|v-t_{2}\right|^{\beta}, \\
\left|\psi_{i}^{0}\left(t_{1}^{\prime}, t_{2}^{\prime}\right)\right| \leq M\|\varphi\|_{\beta}\left|u-t_{1}^{\prime}\right|^{\beta}, \\
\left|\psi_{i}^{0}\left(t_{1}^{\prime}, t_{2}^{\prime}\right)\right| \leq M\|\varphi\|_{\beta}\left|v-t_{2}^{\prime}\right|^{\beta}, \\
i=1,2,3, \\
\left|\psi_{4}^{0}\left(t_{1}, t_{2}\right)\right| \leq M\|\varphi\|_{\beta}, \\
\left|\psi_{4}^{0}\left(t_{1}^{\prime}, t_{2}^{\prime}\right)\right| \leq M\|\varphi\|_{\beta},
\end{gathered}
$$


we obtain that

$$
\begin{aligned}
& \left|E_{m}\left(u, t_{1}\right) d \sigma_{m}(u) \psi_{1}^{0}\left(t_{1}, t_{2}\right) d \sigma_{k}(v) E_{k}\left(v, t_{2}\right)\right| \\
& \quad \leq M\|\varphi\|_{\beta} \rho_{01}^{(\beta / 2)-1} d \rho_{01} \rho_{02}^{(\beta / 2)-1} d \rho_{02}, \\
& \quad\left|E_{m}\left(u, t_{1}\right) d \sigma_{m}(u) \psi_{2}^{0}\left(t_{1}, t_{2}\right) \widetilde{d \sigma_{k}(v)} F_{k}\left(v, t_{2}\right)\right| \\
& \quad \leq M\|\varphi\|_{\beta} \rho_{01}^{\beta-1} d \rho_{01} d \rho_{02}, \\
& \quad \leq M\|\varphi\|_{\beta} d \rho_{01} \rho_{02}^{\beta-1} d \rho_{02}, \\
& \quad\left(u, t_{1}\right) \widehat{d \sigma_{m}(u)} \psi_{3}^{0}\left(t_{1}, t_{2}\right) d \sigma_{k}(v) E_{k}\left(v, t_{2}\right) \mid \\
& \quad \leq M\|\varphi\|_{\beta} d \rho_{01} d \rho_{02} .
\end{aligned}
$$

Thus we have

$$
\begin{aligned}
& \left|R\left(\partial \Omega_{11} \times \partial \Omega_{21}\right)\right| \\
& \quad \leq \sum_{i=1}^{4}\left|\overline{X_{i}}\left(\partial \Omega_{11} \times \partial \Omega_{21}\right)\right|+\sum_{i=1}^{4}\left|\overline{\overline{X_{i}}}\left(\partial \Omega_{11} \times \partial \Omega_{21}\right)\right| \\
& \quad \leq B_{2}\|\varphi\|_{\beta}\left|\left(t_{1}, t_{2}\right)-\left(t_{1}^{\prime}, t_{2}^{\prime}\right)\right|^{\beta} .
\end{aligned}
$$
have

Noting that $\left|v-t_{2}^{\prime}\right| \geq 2 \delta_{2},\left|v-t_{2}\right| \geq 3 \delta_{2}>0$ on $\partial \Omega_{22}$, we

$$
\left|R\left(\partial \Omega_{11} \times \partial \Omega_{22}\right)\right| \leq B_{3}\|\varphi\|_{\beta}\left|\left(t_{1}, t_{2}\right)-\left(t_{1}^{\prime}, t_{2}^{\prime}\right)\right|^{\beta} .
$$

Similarly, we can obtain $\left|R\left(\partial \Omega_{12} \times \partial \Omega_{21}\right)\right| \leq$ $B_{4}\|\varphi\|_{\beta}\left|\left(t_{1}, t_{2}\right)-\left(t_{1}^{\prime}, t_{2}^{\prime}\right)\right|^{\beta}$.

Next we want to prove

$$
\left|R\left(\partial \Omega_{12} \times \partial \Omega_{22}\right)\right| \leq C_{4}\|\varphi\|_{\beta}\left|\left(t_{1}, t_{2}\right)-\left(t_{1}^{\prime}, t_{2}^{\prime}\right)\right|^{\beta} .
$$

According to (33), we have

$$
\begin{aligned}
& \overline{X_{1}}\left(\partial \Omega_{12} \times \partial \Omega_{22}\right)-\overline{\overline{X_{1}}}\left(\partial \Omega_{12} \times \partial \Omega_{22}\right) \\
& =\lambda \int_{\partial \Omega_{12} \times \partial \Omega_{22}}\left[E_{m}\left(u, t_{1}\right)-E_{m}\left(m, t_{1}^{\prime}\right)\right] d \sigma_{m}(u) \psi_{1}^{0}\left(t_{1}, t_{2}\right) \\
& \times d \sigma_{k}(v) E_{k}\left(v, t_{2}\right) \\
& +\lambda \int_{\partial \Omega_{12} \times \partial \Omega_{22}} E_{m}\left(u, t_{1}^{\prime}\right) d \sigma_{m}(u) \psi_{1}^{0}\left(t_{1}, t_{2}\right) d \sigma_{k}(v) \\
& \times\left[E_{k}\left(v, t_{2}\right)-E_{k}\left(v, t_{2}^{\prime}\right)\right] \\
& +\lambda \int_{\partial \Omega_{12} \times \partial \Omega_{22}} E_{m}\left(u, t_{1}^{\prime}\right) d \sigma_{m}(u) \\
& \times\left[\psi_{1}^{0}\left(t_{1}, t_{2}\right)-\psi_{1}^{0}\left(t_{1}^{\prime}, t_{2}^{\prime}\right)\right] \\
& \times d \sigma_{k}(v) E_{k}\left(v, t_{2}^{\prime}\right) \\
& =E_{1}+E_{2}+E_{3} .
\end{aligned}
$$

Similarly, we can deal with

$$
\begin{aligned}
& \overline{X_{2}}\left(\partial \Omega_{12} \times \partial \Omega_{22}\right)-\overline{\overline{X_{2}}}\left(\partial \Omega_{12} \times \partial \Omega_{22}\right)=F_{1}+F_{2}+F_{3}, \\
& \overline{X_{3}}\left(\partial \Omega_{12} \times \partial \Omega_{22}\right)-\overline{\overline{X_{3}}}\left(\partial \Omega_{12} \times \partial \Omega_{22}\right)=G_{1}+G_{2}+G_{3}, \\
& \overline{X_{4}}\left(\partial \Omega_{12} \times \partial \Omega_{22}\right)-\overline{\overline{X_{4}}}\left(\partial \Omega_{12} \times \partial \Omega_{22}\right)=H_{1}+H_{2}+H_{3} .
\end{aligned}
$$

From Lemma 11 and (35) and by $\left|v-t_{2}\right| \geq 3 \delta_{2}>$ $0,\left|v-t_{2}^{\prime}\right| \geq 2 \delta_{2}$, we obtain $\left|E_{2}\right| \leq C_{1}\|\varphi\|_{\beta}\left|\left(t_{1}, t_{2}\right)-\left(t_{1}^{\prime}, t_{2}^{\prime}\right)\right|^{\beta}$. Similarly, we can get the inequality estimation for $E_{1}$ and $G_{2}$. By (36) and (37), $\left|v-t_{2}\right| \geq 3 \delta_{2}>0,\left|v-t_{2}^{\prime}\right| \geq 2 \delta_{2}$, we have $\left|F_{2}\right| \leq C_{2}\|\varphi\|_{\beta}\left|\left(t_{1}, t_{2}\right)-\left(t_{1}^{\prime}, t_{2}^{\prime}\right)\right|^{\beta}$. Similarly, we can obtain the inequality estimation for $F_{1}, G_{1}, H_{1}$, and $H_{2}$.

Since

$$
\begin{aligned}
E_{3}+ & F_{3}+G_{3}+H_{3} \\
= & \frac{1}{2} \lambda_{1} \int_{\partial \Omega_{12}} E_{m}\left(u, t_{1}^{\prime}\right) d \sigma_{m}(u)\left[\varphi\left(u, t_{2}^{\prime}\right)-\varphi\left(u, t_{2}\right)\right] \\
& \left.-\frac{1}{2} \lambda_{1} \int_{\partial \Omega_{12}} F_{m}\left(u, t_{1}^{\prime}\right) \widehat{d \sigma_{m}(u)}\left[\widehat{\varphi\left(u, t_{2}^{\prime}\right.}\right)-\widehat{\varphi\left(u, t_{2}\right)}\right] \\
& +\frac{1}{2} \lambda_{2} \int_{\partial \Omega_{22}}\left[\varphi\left(t_{1}^{\prime}, v\right)-\varphi\left(t_{1}, v\right)\right] d \sigma_{k}(v) E_{k}\left(v, t_{2}^{\prime}\right) \\
& -\frac{1}{2} \lambda_{2} \int_{\partial \Omega_{22}}\left[\widetilde{\varphi\left(t_{1}^{\prime}, v\right)}-\widetilde{\varphi\left(t_{1}, v\right)}\right] \widetilde{d \sigma_{k}(v)} F_{k}\left(v, t_{2}^{\prime}\right) \\
& +\frac{1}{4} \varphi\left(t_{1}, t_{2}\right)-\frac{1}{4} \varphi\left(t_{1}^{\prime}, t_{2}^{\prime}\right),
\end{aligned}
$$

by (35)-(38), we have $\left|E_{3}+F_{3}+G_{3}+H_{3}\right| \leq$ $C_{3}\|\varphi\|_{\beta}\left|\left(t_{1}, t_{2}\right)-\left(t_{1}^{\prime}, t_{2}^{\prime}\right)\right|^{\beta}$.

Summarizing the above discussion shows that

$$
\left|R\left(\partial \Omega_{12} \times \partial \Omega_{22}\right)\right| \leq C_{4}\|\varphi\|_{\beta}\left|\left(t_{1}, t_{2}\right)-\left(t_{1}^{\prime}, t_{2}^{\prime}\right)\right|^{\beta} .
$$

Thus we infer

$$
\left|R\left(\partial \Omega_{1} \times \partial \Omega_{2}\right)\right| \leq C_{5}\|\varphi\|_{\beta}\left|\left(t_{1}, t_{2}\right)-\left(t_{1}^{\prime}, t_{2}^{\prime}\right)\right|^{\beta} .
$$

Hence

$$
\left\|\sum_{i=1}^{4} X_{i}\left(t_{1}, t_{2}\right)\right\|_{\beta} \leq J_{4}\|\varphi\|_{\beta} .
$$

This completes the proof. 
Corollary 14. If $\varphi\left(t_{1}, t_{2}\right) \in H\left(\partial \Omega_{1} \times \partial \Omega_{2}, \beta\right)$, then

$$
\begin{array}{ll}
\left\|\phi^{++}\left(t_{1}, t_{2}\right)\right\|_{\beta} \leq J_{5}\|\varphi\|_{\beta}, & \left\|\phi^{+-}\left(t_{1}, t_{2}\right)\right\|_{\beta} \leq J_{5}\|\varphi\|_{\beta}, \\
\left\|\phi^{-+}\left(t_{1}, t_{2}\right)\right\|_{\beta} \leq J_{5}\|\varphi\|_{\beta}, & \left\|\phi^{--}\left(t_{1}, t_{2}\right)\right\|_{\beta} \leq J_{5}\|\varphi\|_{\beta} .
\end{array}
$$

Theorem 15. Let $A(x, y), B(x, y), C(x, y), D(x, y), g(x, y) \in$ $H\left(\partial \Omega_{1} \times \partial \Omega_{2}, \beta\right)$; then the function $f\left(t_{1}, t_{2}, \phi^{1}, \phi^{2}, \phi^{3}, \phi^{4}\right)$ is a Hölder continuous function for $\left(t_{1}, t_{2}\right) \in \partial \Omega_{1} \times \partial \Omega_{2}$ and satisfies the Lipschitz-condition for $\phi^{1}, \phi^{2}, \phi^{3}, \phi^{4}$ and any $\left(t_{1}, t_{2}\right)$, namely,

$$
\begin{aligned}
& \left|f\left(t_{11}, t_{21}, \phi_{1}^{1}, \phi_{1}^{2}, \phi_{1}^{3}, \phi_{1}^{4}\right)-f\left(t_{12}, t_{22}, \phi_{2}^{1}, \phi_{2}^{2}, \phi_{2}^{3}, \phi_{2}^{4}\right)\right| \\
& \leq J_{6}\left|\left(t_{11}, t_{21}\right)-\left(t_{12}, t_{22}\right)\right|^{\beta}+J_{7}\left|\phi_{1}^{1}-\phi_{2}^{1}\right| \\
& \quad+J_{8}\left|\phi_{1}^{2}-\phi_{2}^{2}\right|+J_{9}\left|\phi_{1}^{3}-\phi_{2}^{3}\right|+J_{10}\left|\phi_{1}^{4}-\phi_{2}^{4}\right|,
\end{aligned}
$$

where $J_{i}(i=6, \ldots, 10)$ is a positive constant and has nothing to do with $t_{1 j}, t_{2 j}, \phi_{j}^{1}, \ldots, \phi_{j}^{4}, j=1$, 2. If $f(0,0,0,0,0,0)=$ $0,\|A+B\|_{\beta}<\varepsilon,\|C+D\|_{\beta}<\varepsilon,\|D+B\|_{\beta}<\varepsilon,\|1-4 B\|_{\beta}<$ $\varepsilon, 0<\varepsilon<1,0<\mu=\varepsilon J_{0}\left(2 J_{5}+J_{2}+1\right)<1,\|g\|_{\beta}<\delta, 0<\delta<$ $M(1-\mu) / 4 \cdot J_{0}\left(J_{13}+J_{14} M\right)$, then the problem $R$ has at least one solution, where $M\left(\|\varphi\|_{\beta}<M\right), J_{13}, J_{14}$ are both positive constants satisfying $\|f\|_{\beta} \leq J_{13}+J_{14}\|\varphi\|_{\beta}$.

Proof. Suppose that $T=\left\{\varphi \mid \varphi \in H\left(\partial \Omega_{1} \times \partial \Omega_{2}, \beta\right),\|\varphi\|_{\beta}<\right.$ $M\}$ be denoted a subset of $C\left(\partial \Omega_{1} \times \partial \Omega_{2}\right)$. From Theorem 13, Corollary 14, and (38), we obtain $C\left(f, \partial \Omega_{1} \times \partial \Omega_{2}\right) \leq J_{11}+$ $J_{12}\|\varphi\|_{\beta}$. Similarly, we can get $\|f\|_{\beta} \leq J_{13}+J_{14}\|\varphi\|_{\beta}$. Hence, by (12) and $F \varphi=\varphi,\|F \varphi\|_{\beta} \leq M$ is derived. This shows that the operator $F$ is the mapping of $T \rightarrow T$.

Next we prove the $F$ is a continuous mapping.

Suppose that the sequence of functions $\left\{\varphi_{n}\right\} \in T$ uniformly converges to a function $\varphi\left(t_{1}, t_{2}\right),\left(t_{1}, t_{2}\right) \in \partial \Omega_{1} \times$ $\partial \Omega_{2}$; thus for arbitrary $\varepsilon>0$ and if $n$ is large enough, then $\left|\left(P_{i}+Q_{i}\right) \varphi_{n}-\left(P_{i}+Q_{i}\right) \varphi\right|<\varepsilon,(i=1,2)$.

Now we consider $P_{3} \varphi_{n}-P_{3} \varphi$ by

$$
\begin{aligned}
P_{3} \varphi_{n} & -P_{3} \varphi \\
= & \sum_{i=1}^{2} A_{i}\left(\partial \Omega_{1} \times \partial \Omega_{2}\right)+\sum_{i=1}^{2} B_{i}\left(\partial \Omega_{1} \times \partial \Omega_{2}\right) \\
& +\sum_{i=1}^{2} C_{i}\left(\partial \Omega_{1} \times \partial \Omega_{2}\right)+\sum_{i=1}^{2} D_{i}\left(\partial \Omega_{1} \times \partial \Omega_{2}\right)+\sum_{i=1}^{3} E_{i},
\end{aligned}
$$

where

$$
\begin{gathered}
A_{1}\left(\partial \Omega_{1} \times \partial \Omega_{2}\right) \\
\quad=4 \lambda \int_{\partial \Omega_{1} \times \partial \Omega_{2}} E_{m}\left(u, t_{1}\right) d \sigma_{m}(u) \psi_{1 n}(u, v) d \sigma_{k}(v) \\
\quad \times E_{k}\left(v, t_{2}\right),
\end{gathered}
$$

$$
\begin{gathered}
A_{2}\left(\partial \Omega_{1} \times \partial \Omega_{2}\right) \\
\quad=-4 \lambda \int_{\partial \Omega_{1} \times \partial \Omega_{2}} E_{m}\left(u, t_{1}\right) d \sigma_{m}(u) \psi_{1}(u, v) d \sigma_{k}(v) \\
\quad \times E_{k}\left(v, t_{2}\right),
\end{gathered}
$$

$B_{1}\left(\partial \Omega_{1} \times \partial \Omega_{2}\right)$

$$
\begin{gathered}
=-4 \lambda \int_{\partial \Omega_{1} \times \partial \Omega_{2}} E_{m}\left(u, t_{1}\right) d \sigma_{m}(u) \psi_{2 n}(u, v) \widetilde{d \sigma_{k}(v)} \\
\times F_{k}\left(v, t_{2}\right),
\end{gathered}
$$

$B_{2}\left(\partial \Omega_{1} \times \partial \Omega_{2}\right)$

$$
\begin{gathered}
=4 \lambda \int_{\partial \Omega_{1} \times \partial \Omega_{2}} E_{m}\left(u, t_{1}\right) d \sigma_{m}(u) \psi_{2}(u, v) \widetilde{d \sigma_{k}(v)} \\
\times F_{k}\left(v, t_{2}\right)
\end{gathered}
$$

$C_{1}\left(\partial \Omega_{1} \times \partial \Omega_{2}\right)$

$=-4 \lambda \int_{\partial \Omega_{1} \times \partial \Omega_{2}} F_{m}\left(u, t_{1}\right) \widehat{d \sigma_{m}(u)} \psi_{3 n}(u, v) d \sigma_{k}(v)$

$$
\times E_{k}\left(v, t_{2}\right),
$$

$$
\begin{gathered}
C_{2}\left(\partial \Omega_{1} \times \partial \Omega_{2}\right) \\
=4 \lambda \int_{\partial \Omega_{1} \times \partial \Omega_{2}} F_{m}\left(u, t_{1}\right) \widehat{d \sigma_{m}(u)} \psi_{3}(u, v) d \sigma_{k}(v) \\
\times E_{k}\left(v, t_{2}\right),
\end{gathered}
$$

$D_{1}\left(\partial \Omega_{1} \times \partial \Omega_{2}\right)$

$=4 \lambda \int_{\partial \Omega_{1} \times \partial \Omega_{2}} F_{m}\left(u, t_{1}\right) \widehat{d \sigma_{m}(u)} \psi_{4 n} \widetilde{d \sigma_{k}(v)} F_{k}\left(v, t_{2}\right)$,

$$
\begin{aligned}
D_{2} & \left(\partial \Omega_{1} \times \partial \Omega_{2}\right) \\
= & -4 \lambda \int_{\partial \Omega_{1} \times \partial \Omega_{2}} F_{m}\left(u, t_{1}\right) \widehat{d \sigma_{m}(u)} \psi_{4} \widetilde{d \sigma_{k}(v)} F_{k}\left(v, t_{2}\right),
\end{aligned}
$$

$$
\begin{gathered}
E_{1}=\varphi\left(t_{1}, t_{2}\right)-\varphi_{n}\left(t_{1}, t_{2}\right), \\
E_{2}=\left(P_{1}+Q_{1}\right) \varphi_{n}-\left(P_{1}+Q_{1}\right) \varphi, \\
E_{3}=\left(P_{2}+Q_{2}\right) \varphi_{n}-\left(P_{2}+Q_{2}\right) \varphi .
\end{gathered}
$$


Suppose $6 \delta<d_{i}, i=1,2, \delta>0, O\left(\left(t_{1}, t_{2}\right), 3 \delta\right)$ is the $3 \delta$-neighborhood of $\left(t_{1}, t_{2}\right)$ with the center at point $\left(t_{1}, t_{2}\right) \in$ $\partial \Omega_{1} \times \partial \Omega_{2}$ and the radius $3 \delta, \partial \Omega_{i 1} \times \partial \Omega_{i 2}$ is as above; then

$$
\begin{aligned}
& \square_{j}\left(\partial \Omega_{1} \times \partial \Omega_{2}\right) \\
& =\square_{j}\left(\partial \Omega_{11} \times \partial \Omega_{21}\right)+\square_{j}\left(\partial \Omega_{11} \times \partial \Omega_{22}\right) \\
& +\square_{j}\left(\partial \Omega_{12} \times \partial \Omega_{21}\right)+\square_{j}\left(\partial \Omega_{12} \times \partial \Omega_{22}\right), \\
& (\square=A, B, C, D, j=1,2) .
\end{aligned}
$$

By (35), we can obtain that

$$
\begin{gathered}
\left|A_{1}\left(\partial \Omega_{11} \times \partial \Omega_{21}\right)\right| \leq J_{15} \delta^{\beta} \leq J_{16} \delta^{\beta / 2} \\
\left|A_{1}\left(\partial \Omega_{12} \times \partial \Omega_{21}\right)\right| \leq J_{17} \delta^{\beta / 2} \\
\left|A_{1}\left(\partial \Omega_{11} \times \partial \Omega_{22}\right)\right| \leq J_{18} \delta^{\beta / 2}
\end{gathered}
$$

Similarly, we can get the inequality estimations for $A_{2}\left(\partial \Omega_{11} \times\right.$ $\left.\partial \Omega_{21}\right), A_{2}\left(\partial \Omega_{12} \times \partial \Omega_{21}\right)$, and $A_{2}\left(\partial \Omega_{11} \times \partial \Omega_{22}\right)$. By (35), (36), and (37), we can obtain the similar inequality estimations for $B_{i}\left(\partial \Omega_{11} \times \partial \Omega_{21}\right), B_{i}\left(\partial \Omega_{12} \times \partial \Omega_{21}\right), B_{i}\left(\partial \Omega_{11} \times \partial \Omega_{22}\right), C_{i}\left(\partial \Omega_{11} \times\right.$ $\left.\partial \Omega_{21}\right), C_{i}\left(\partial \Omega_{12} \times \partial \Omega_{21}\right), C_{i}\left(\partial \Omega_{11} \times \partial \Omega_{22}\right), D_{i}\left(\partial \Omega_{11} \times \partial \Omega_{21}\right)$, $D_{i}\left(\partial \Omega_{12} \times \partial \Omega_{21}\right)$, and $D_{i}\left(\partial \Omega_{11} \times \partial \Omega_{22}\right), i=1,2$, respectively.

From

$$
\begin{gathered}
A_{1}\left(\partial \Omega_{12} \times \partial \Omega_{22}\right)+A_{2}\left(\partial \Omega_{12} \times \partial \Omega_{22}\right) \\
=4 \lambda \int_{\partial \Omega_{12} \times \partial \Omega_{22}} E_{m}\left(u, t_{1}\right) d \sigma_{m}(u) W_{1}(u, v) d \sigma_{k}(v) \\
\times E_{k}\left(v, t_{2}\right)
\end{gathered}
$$

where

$$
\begin{aligned}
W_{1}(u, v) \\
=\left\{\left[\varphi_{n}(u, v)-\varphi(u, v)\right]-\left[\varphi_{n}\left(t_{1}, v\right)-\varphi\left(t_{1}, v\right)\right]\right\} \\
\quad+\left\{\left[\varphi_{n}\left(t_{1}, t_{2}\right)-\varphi\left(t_{1}, t_{2}\right)\right]-\left[\varphi_{n}\left(u, t_{2}\right)-\varphi\left(u, t_{2}\right)\right\},\right.
\end{aligned}
$$

since $\left|W_{1}(u, v)\right| \leq 2\left\|\varphi_{n}-\varphi\right\|_{\beta}\left|u-t_{1}\right|^{\beta / 2}\left|v-t_{2}\right|^{\beta / 2}$ and from (35), we have

$$
\left|A_{1}\left(\partial \Omega_{12} \times \partial \Omega_{22}\right)+A_{2}\left(\partial \Omega_{12} \times \partial \Omega_{22}\right)\right| \leq J_{19}\left\|\varphi_{n}-\varphi\right\|_{\beta} .
$$

Similarly, we can get the inequality estimations for $B_{1}\left(\partial \Omega_{12} \times\right.$ $\left.\partial \Omega_{22}\right)+B_{2}\left(\partial \Omega_{12} \times \partial \Omega_{22}\right), C_{1}\left(\partial \Omega_{12} \times \partial \Omega_{22}\right)+C_{2}\left(\partial \Omega_{12} \times \partial \Omega_{22}\right)$.

From

$$
\begin{aligned}
& D_{1}\left(\partial \Omega_{12} \times \partial \Omega_{22}\right)+D_{2}\left(\partial \Omega_{12} \times \partial \Omega_{22}\right) \\
& =4 \lambda \int_{\partial \Omega_{12} \times \partial \Omega_{22}} F_{m}\left(u, t_{2}\right) \widehat{d \sigma_{m}(u)} W_{4}(u, v) \widetilde{d \sigma_{k}(v)} F_{k}\left(v, t_{2}\right)
\end{aligned}
$$

where

$$
\begin{aligned}
& W_{4}(u, v) \\
& =\left\{\left[\widetilde{\widetilde{\varphi_{n}(u, v)}}-\widetilde{\widehat{\varphi(u, v)}}\right]-\left[\widetilde{\varphi_{n}\left(t_{1}, v\right)}-\widetilde{\varphi\left(t_{1}, v\right)}\right]\right\} \\
& \left.+\left\{\left[\varphi_{n}\left(t_{1}, t_{2}\right)-\varphi\left(t_{1}, t_{2}\right)\right]-\left[\widehat{\varphi_{n}\left(u, t_{2}\right.}\right)-\widehat{\varphi\left(u, t_{2}\right)}\right]\right\},
\end{aligned}
$$

since $\left|W_{4}(u, v)\right| \leq 4\left\|\varphi_{n}-\varphi\right\|_{\beta}$ and from (38), we obtain that

$$
\left|D_{1}\left(\partial \Omega_{12} \times \partial \Omega_{22}\right)+D_{2}\left(\partial \Omega_{12} \times \partial \Omega_{22}\right)\right| \leq J_{21}\left\|\varphi_{n}-\varphi\right\|_{\beta} .
$$

Summarizing the above discussion, we conclude $\mid P_{3} \varphi_{n}-$ $P_{3} \varphi \mid \leq J_{22}\left(\varepsilon+\delta^{\beta / 2}+\left\|\varphi_{n}-\varphi\right\|_{\beta}\right)$. Then for arbitrary $\varepsilon>0$, we first choose a sufficiently small number $\delta$ and next select a sufficiently large positive integer $n$; we have

$$
\left|P_{3} \varphi_{n}-P_{3} \varphi\right|<G \varepsilon
$$

where $G$ is a positive constant.

Finally, we can choose $n$ large enough such that $\mid F \varphi_{n}-$ $F \varphi \mid<W \varepsilon(W$ is a positive constant). Hence we can obtain $F$ : $T \rightarrow T$ is a continuous mapping. According to Ascoli-Arzela Theorem, $T$ is a compact set in the space $C\left(\partial \Omega_{1} \times \partial \Omega_{2}\right)$. Based on the Schauder fixed point principle, there exists a function $\varphi \in H\left(\partial \Omega_{1} \times \partial \Omega_{2}, \beta\right)$ satisfying the equation $F \varphi=\varphi$.

Corollary 16. If $f \equiv 1$ in Theorem 15 , then the Problem $R$ has the unique solution.

Proof. This corollary is not difficult to verify by the contraction mapping principle when $f \equiv 1$ in Theorem 15 .

\section{Conflict of Interests}

The authors declare that there is no conflict of interests regarding the publication of this paper.

\section{Acknowledgment}

This work was completed with the support of the National Natural Science Foundation of China (NNSFC) through Grants no. 10671207 and no. 11301136.

\section{References}

[1] F. Brackx, R. Delanghe, and F. Sommen, "Clifford analysis," in Research Note in Mathematics, vol. 76, Pitman Book, London, UK, 1982.

[2] Z. Z. Xu, "Riemann boundary value problem for regular function in Clifford algebra," Chinese Science Bulletin, vol. 32, pp. 476-477, 1987.

[3] Z. Z. Xu, "On linear and nonlinear Riemann-Hilbert problems for regular function with values in a Clifford algebra," Chinese Annals of Mathematics B, vol. 11, no. 3, pp. 349-358, 1990.

[4] G. C. Wen, Clifford Annalysis and Elliptic System, Hyperbolic Systems of First Order Equations, World Scientific Publishing, Singapore, 1991. 
[5] S. Huang, "Nonlinear boundary value problem for biregular functions in Clifford analysis," Science in China A, vol. 39, no. 11, pp. 1152-1163, 1996.

[6] S. Huang, Y. Y. Qiao, and G. C. Wen, Real and Complex Clifford Analysis, Springer, New York, NY, USA, 2005.

[7] Y. Y. Qiao, "A nonlinear boundary value problem with a Haseman shift for biregular function," Journal of Systems Science and Mathematical Sciences, vol. 19, no. 4, pp. 484-489, 1999.

[8] Y. Y. Qiao, "A nonlinear boundary value problem with a shift for generalized biregular functions," Journal of Systems Science and Mathematical Sciences, vol. 22, no. 1, pp. 43-49, 2002.

[9] Y. Y. Qiao, "A boundary value problem for hypermonogenic functions in Clifford analysis," Science in China A: Mathematics, vol. 48, pp. 324-332, 2005.

[10] S.-L. Eriksson and H. Leutwiler, "Hypermonogenic functions," in Clifford Algebras and Their Applications in Mathematical Physics, vol. 2, pp. 287-302, Birkhäauser, Boston, Mas, USA, 2000.

[11] S.-L. Eriksson, "Integral formulas for hypermonogenic functions," Bulletin of the Belgian Mathematical Society, vol. 11, no. 5, pp. 705-718, 2004.

[12] S. L. Eriksson and H. Leutwiler, "An improved Cauchy formula for hypermonogenic functions," Advances in Applied Clifford Algebras, vol. 19, no. 2, pp. 269-282, 2009.

[13] Z. Z. Zhang and J. Y. Du, "On certain Riemann boundary value problems and singular integral equations in Clifford analysis," Chinese Journal of Contemporary Mathematics, vol. 22, pp. 237244, 2001.

[14] Z. Z. Zhang and J. Y. Du, "Laurent expansion and residue theorems in universal Clifford analysis," Acta Mathematica Scientia A, vol. 23, no. 6, pp. 692-703, 2003.

[15] X. L. Bian, S.-L. Eriksson, J. Li, and Y. Y. Qiao, "Cauchy integral formula and Plemelj formula of bihypermonogenic functions in real Clifford analysis," Complex Variables and Elliptic Equations, vol. 54, no. 10, pp. 957-976, 2009.

[16] H. J. Yang, Y. H. Xie, and Y. Y. Qiao, "A kind of boundary value problem for hypermonogenic function vectors," Journal of Mathematical Research and Exposition, vol. 31, no. 3, pp. 490496, 2011.

[17] Z. Z. Zhang and K. Gürlebeck, "Some Riemann boundary value problems in Clifford analysis (I)," Complex Variables and Elliptic Equations, vol. 58, no. 7, pp. 991-1003, 2013. 


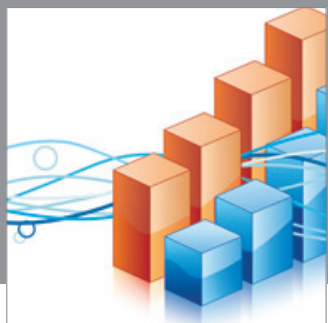

Advances in

Operations Research

mansans

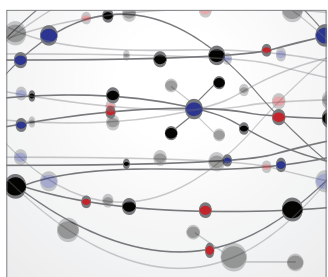

The Scientific World Journal
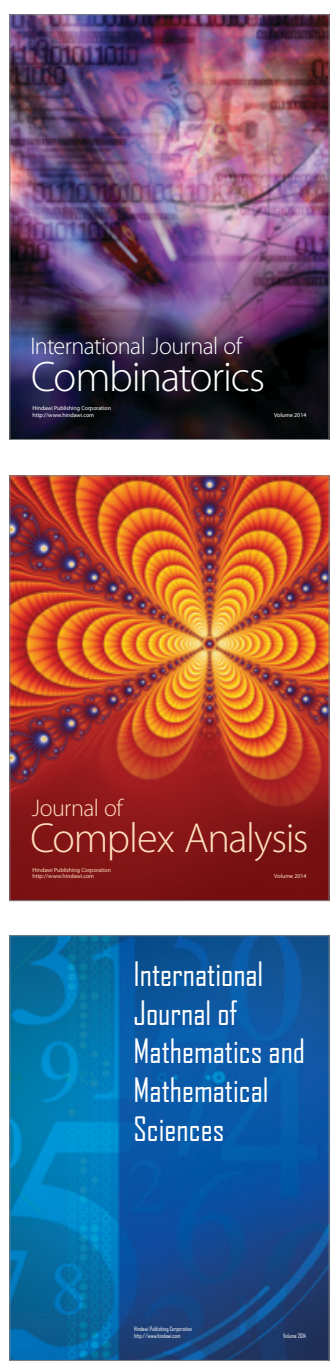
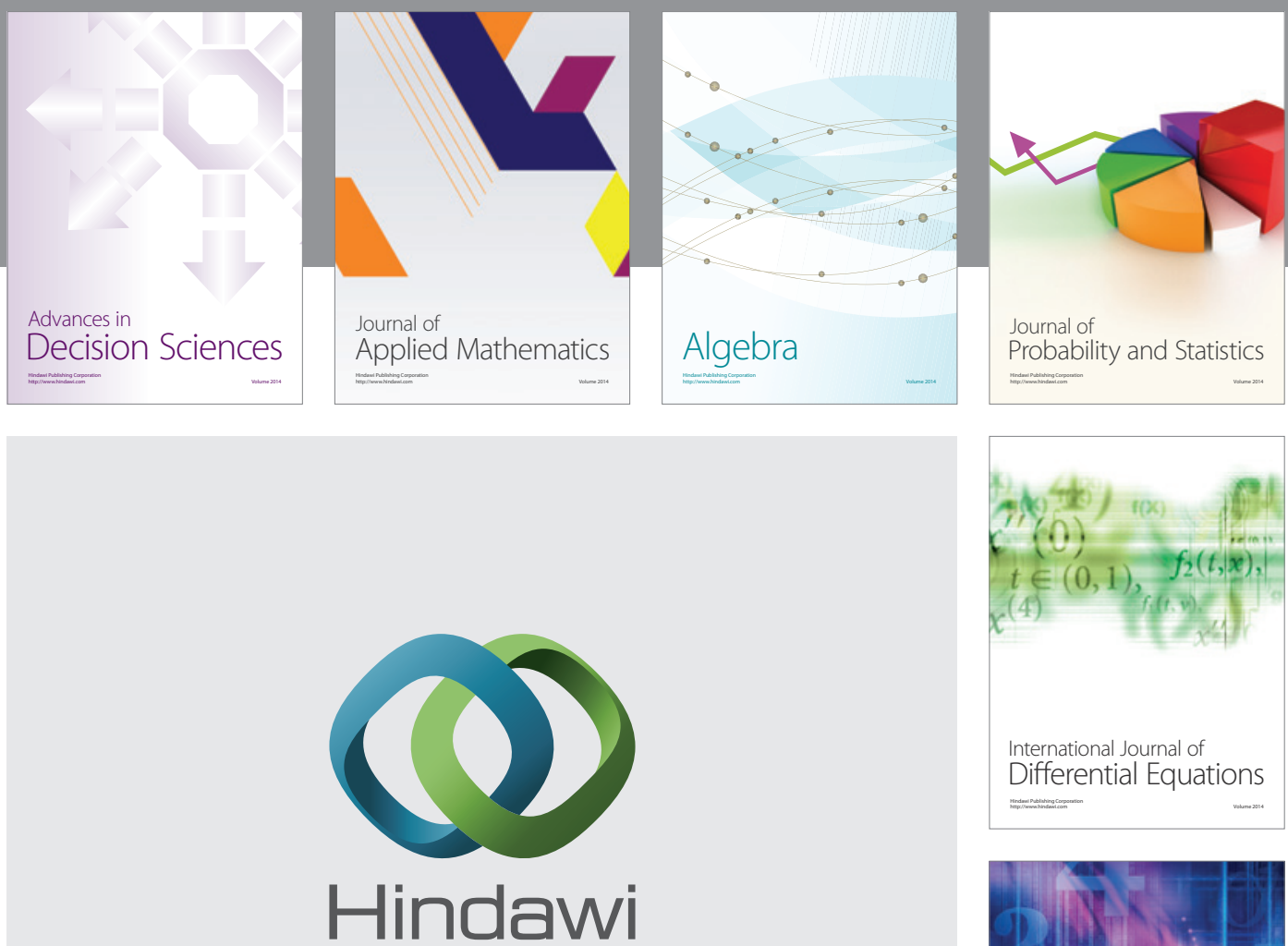

Submit your manuscripts at http://www.hindawi.com
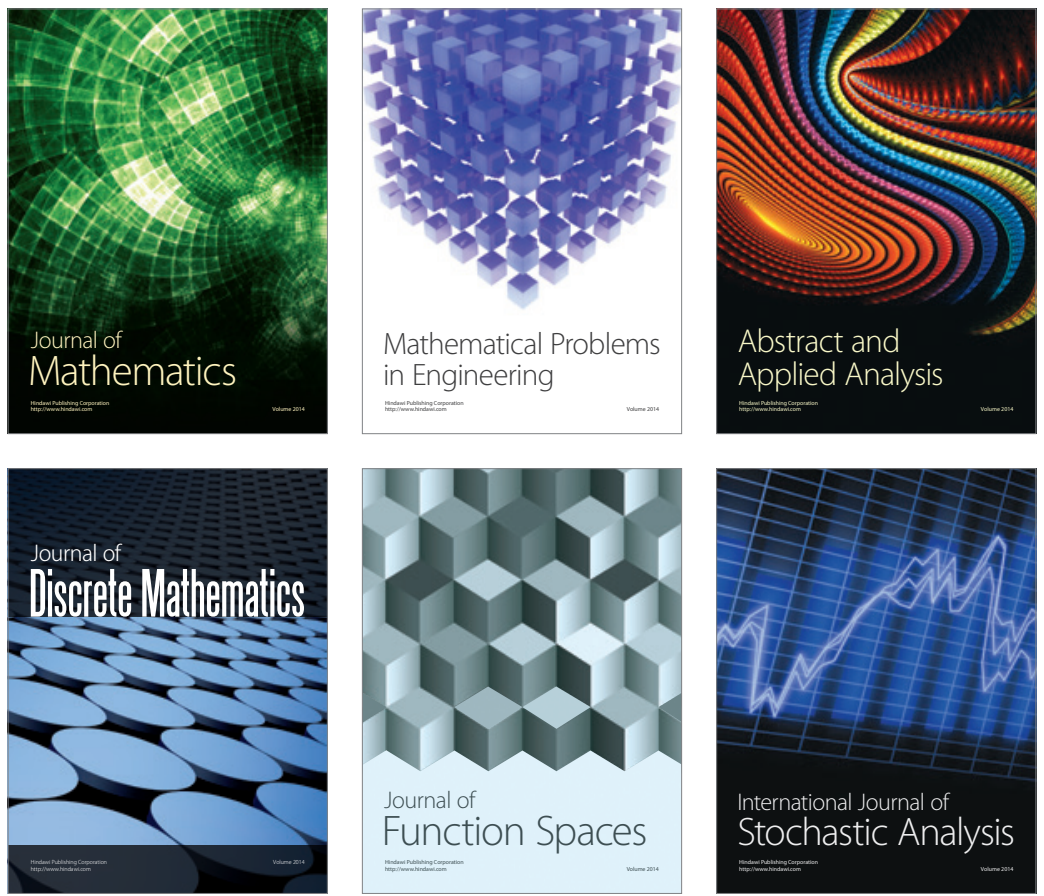

Journal of

Function Spaces

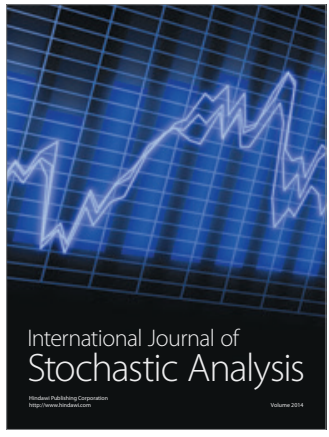

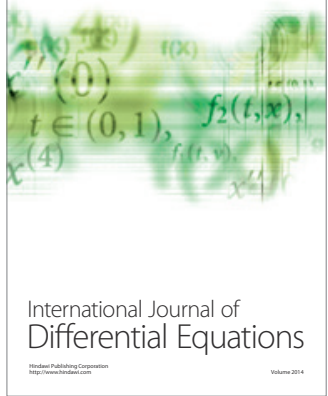
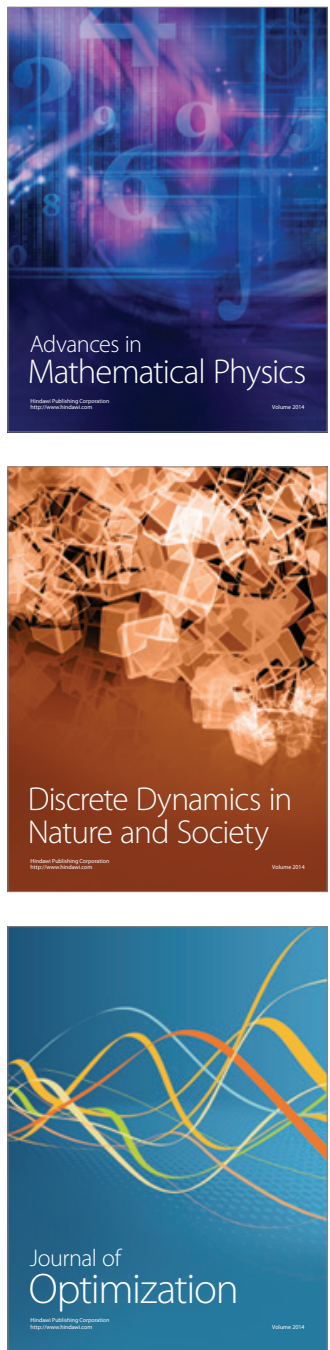